\title{
Proporção de Doenças Musculoesqueléticas em Membros Inferiores nos Integrantes da Polícia Militar do Estado da Bahia
}

\author{
Daniela Alves Silva \\ Acadêmica do 5o ano do curso de Fisioterapia da Escola \\ Bahiana de Medicina e Saúde Pública \\ (danieella@gmail.com) \\ Acadêmica do $5^{\circ}$ ano do curso de Fisioterapia da Escola \\ Vanessa Santos Lima \\ Bahiana de Medicina e Saúde Pública \\ (vanessalima.fisioterapiadf@gmail.com) \\ Professora Adjunta do Curso de Fisioterapia da Escola \\ Bahiana de Medicina e Saúde Pública; Esp. em Correção da \\ Postura e Tratamento da Dor; Membro participante do \\ Ana Lúcia Barbosa Góes \\ Projeto Desenvolvimento e Fortalecimento de Pesquisas em \\ Saúde e Ocupação nas Forças Armadas do Brasil - Instituto \\ de Saúde Coletiva/ Universidade Federal da Bahia \\ (albgoes@bahiana.edu.br) \\ Trabalho de conclusão de curso de fisioterapia da Escola \\ Bahiana de Medicina e Saúde Pública apresentado na Escola \\ Origem da Pesquisa Bahiana de Medicina e Saúde Pública, Av Dom João VI, 275, \\ Brotas, 40 290-000 Salvador-BA, Brasil. Telefone: (71) \\ 3276-8200 / 3276-8261 \\ Tipo de Pesquisa Artigo Original \\ Tipo de Análise do Manuscrito \\ Triple Blind Peer Review \\ Recebido em Dez/ 2011 \\ Aprovado em Dez/ 2011
}

\section{RESUMO}

Introdução: As doenças músculo esqueléticas (DME) são a maior causa de limitação funcional na população adulta e a primeira causa de auxílio-doença no Brasil. 0 trabalho do policial militar (PM) exige a prática de atividades rigorosas, tornando-o susceptível ao desenvolvimento de lesões nos membros inferiores (MMII). Objetivo: Estimar a proporção de lesões músculo esqueléticas na região dos membros inferiores e fatores associados em integrantes da PM do Estado da Bahia. Métodos: Estudo do tipo transversal de série temporal, realizado com 3.652 diagnósticos relacionados à DME em MMII no período de 2003 a 2007. Para associação entre DME, idade, posto, local de trabalho e segmento corporal utilizaram-se proporção e razão de proporção a 95\% de confiança. Resultados: A proporção geral de DME em MMII foi de 27,5\%, a maior proporção para o desenvolvimento das doenças em PM se deu a partir de 26 a 34 anos de idade $(30,50 \%)$, em militares praças $(27,80 \%)$, no setor operativo $(27,8 \%)$, sendo o segmento mais afetado joelhos $(12,6 \%)$. Conclusão: A proporção de DME em MMII em PM foi baixa, tendo como público-alvo praças em plena idade produtiva e que estão na linha de frente de combate. Sugerem-se novos trabalhos evidenciando a problemática em nível nacional, bem como políticas de prevenção, diminuindo as chances de desenvolvimento das lesões.

Palavras-chave: Doença Músculo Esquelética; Policiais Militares; Membros Inferiores; Ocupação; Saúde do Trabalhador 


\title{
Proportion of Musculoskeletal Diseases \\ in the Lower Limbs Members of the Military Police of the State of Bahia
}

\section{ABSTRACT}

Introduction: musculoskeletal diseases (MSDs) are the major cause of functional limitation in adults and the leading cause of sickness in Brazil. The work of the military police (MP) requires the practice of strict, making it susceptible to the development of lesions in the lower limbs (LL). Objective: To estimate the proportion of musculoskeletal disorders in the lower limbs and associated factors among members of the PM of the State of Bahia. Methods: A cross-sectional time series, conducted with 3,652 diagnoses related to the DME in lower limbs during the period 2003 to 2007. For association between DME, age, rank, place of work and body segment were used proportion and aspect ratio to $95 \%$ confidence. Results: The overall proportion of DME in lower limbs was $27.5 \%$, the highest proportion for the development of diseases in PM occurred from 26 to 34 years old (30.50\%), in military courts $(27,80 \%)$, operating in the sector $(27.8 \%)$ being the most affected segment knees $(12.6 \%)$. Conclusion: The proportion of DME in LL and PM were low, with the audience in full squares productive age and who are on the front lines of combat. Suggest new studies showing the problems at the national level as well as prevention policies, reducing the chances of developing ulcers.

Keywords: Skeletal Muscle Disease, Military Police, Leg, Occupation, Occupational Health

\author{
Proporción de Enfermedades Musculoesqueléticas \\ en los Miembros Inferiores de la Policía Militar del Estado de Bahía
}

\section{RESUMEN}

Introducción: Las enfermedades músculo-esqueléticos (TME) constituyen la principal causa de limitación funcional en adultos y la principal causa de la enfermedad en Brasil. El trabajo de la policía militar (PM) requiere la práctica de la estricta, por lo que es susceptible al desarrollo de lesiones en las extremidades inferiores (LL). Objetivo: estimar la proporción de los trastornos musculoesqueléticos en las extremidades inferiores y los factores asociados entre los miembros de la PM del estado de Bahía. Métodos: Una serie de tiempo de corte transversal, realizado con 3.652 diagnósticos relacionados con el DME en las extremidades inferiores durante el período 2003 a 2007. Por asociación entre DME, edad, rango, el lugar de trabajo y segmento del cuerpo se utiliza la proporción y la relación de aspecto de confianza del 95\%. Resultados: La proporción global de DME en las extremidades inferiores fue del $27,5 \%$, la proporción más alta para el desarrollo de enfermedades en los PM se produjo a partir del 26 a 34 años (30,50\%), en los tribunales militares $(27,80 \%)$, que opera en el sector $(27,8 \%)$, siendo las rodillas segmento más afectado (12,6\%). Conclusión: La proporción de DME en la LL y PM fueron bajos, con el público en edad productiva y llena plazas que están en las primeras líneas de combate. Proponer nuevos estudios que muestran los problemas a nivel nacional, así como las políticas de prevención, la reducción de las posibilidades de desarrollar úlceras.

Palabras clave: Enfermedad del músculo esquelético, la Policía Militar, la pierna, Ocupación, Salud Ocupacional

\section{Proportion des maladies musculo-squelettiques dans les membres inférieurs membres de la police militaire de l'État de Bahia}

\section{RÉSUMÉ}

Introduction: les maladies musculo-squelettiques (TMS) sont la principale cause de limitation fonctionnelle chez les adultes et la première cause de maladie au Brésil. Le travail de la police militaire (MP) exige la pratique de stricte, ce qui rend sensible à l'évolution des lésions des 
membres inférieurs (LL). Objectif: estimer la proportion de troubles musculo-squelettiques dans les membres inférieurs et des facteurs associés chez les membres de la PM de l'Etat de Bahia. Méthodes: Une série chronologique transversale, menée avec 3.652 diagnostics liés à la DME dans les membres inférieurs au cours de la période de 2003 à 2007. Pour l'association entre les DME, l'âge, le grade, le lieu de travail et le segment corporel ont été utilisées proportion et l'aspect ratio de confiance de 95\%. Résultats: La proportion globale de DME dans les membres inférieurs était de $27,5 \%$, la plus forte proportion pour le développement de maladies dans les PM a eu lieu du 26 au 34 ans (30,50\%), devant les tribunaux militair es $(27,80 \%)$, opérant dans le secteur $(27,8 \%)$ étant les genoux du segment les plus touchés $(12,6 \%)$. Conclusion: La proportion de DME dans LL et PM étaient faibles, avec le public à l'âge carrés pleine productif et qui sont sur les lignes de front de combat. Proposer de nouvelles études montrant les problèmes au niveau national ainsi que les politiques de prévention, réduire les risques de développer des ulcères.

Mots-clés: maladie du muscle squelettique, la police militaire, Leg, Travail, Santé au travail

\section{Introdução}

As doenças músculo esqueléticas apresentam alta prevalência e impacto, podendo afetar centenas de milhões de pessoas em todo o mundo1.

$\mathrm{Na}$ população trabalhadora brasileira os distúrbios osteomusculares relacionados ao trabalho figuram entre as doenças mais prevalentes, representando um relevante problema de saúde pública, além de serem responsáveis pela maior parte dos afastamentos do trabalho e pelos custos com pagamentos de indenizações, tanto no Brasil como na maior parte dos países industrializados2-4.

Em diversos países, representam a maior causa de recebimento de compensações por dias perdidos no trabalho. No Brasil, em 2006, 48,2\% dos benefícios previdenciários por doença do trabalho foram concedidos por causa das doenças musculoesqueléticas (Ministério da Previdência Social)5.

Tratando-se do contexto do policial militar, este se caracteriza por ser lotado no estado, responsável pela execução da política de segurança pública6. Diante da importância do papel desenvolvido pelo policial militar, é exigido dele um elevado nível de saúde tanto física quanto mental para suportar as cargas impostas no ambiente militar como extensas jornadas de trabalho, problemas ergonômicos, exposição a agentes químicos, físicos e biológicos que, reconhecidamente, são considerados fatores de risco ocupacional para o trabalhador 7, 8.

O PM está envolvido com atividades que exigem principalmente do sistema músculo esquelético - corrida, saltos, prática de esporte, carregamento de peso, marcha, movimentos repetitivos, elevadas cargas e prática de atividades físicas rigorosas 9 - em que, qualquer desordem do mesmo, pode resultar no desenvolvimento de lesões. Sendo assim, atenta-se para a região dos membros inferiores, que por ser composta por grandes grupos musculares, estar envolvida em todas as atividades desenvolvidas pelo policial militar, torna-se a mais exigida e exposta durante suas atividades, podendo ser uma região susceptível para o desenvolvimento de lesões.

O estudo visa contribuir com essa discussão ampliando e enriquecendo a divulgação científica, já que a busca sobre as lesões decorrentes dessa atividade militar permitiu constatar uma escassez de informações e de investigações sobre o assunto, sendo este um campo pouco explorado. Com aprimoramento nessa área será possível diminuir a freqüência de lesões fazendo com que a saúde desses militares não seja prejudicada e que seja menor o número de afastamentos de militares dos seus postos de trabalho, consequentemente 
diminuindo os custos com a concessão de benefícios por licença de trabalho ou aposentadoria por invalidez.

A partir dessas considerações, bem como da escassez de pesquisas no Brasil sobre o tema, este estudo teve como objetivo estimar a proporção de doença músculo esquelética em membros inferiores em Policiais Militares do Estado da Bahia, verificando a faixa etária, a hierarquia, o posto e o segmento de maior ocorrência dessas lesões.

\section{Material \& Método}

Trata-se de um estudo transversal de série temporal realizado com todos os militares da Bahia que procuraram o serviço de saúde no período de Janeiro de 2003 à Dezembro de 2007. É um estudo baseado em dados secundários obtidos a partir de documentos relacionados à inspeção de saúde, fornecidos pela junta de saúde da Polícia Militar do Estado da Bahia, e sua coleta se deu para a realização de estudo prévio sobre Lombalgia no ano de 200910.

Os dados presentes no banco foram categorizados da seguinte forma: Idade (19-25 anos, 26-34 anos e acima de 35 anos), posto (praça - soldado, cabo, terceiro sargento, segundo sargento, primeiro sargento, sub-tenente, aspirante e oficial - segundo tenente, primeiro tenente, capitão, major, tenente-coronel, coronel), lesão de membros inferiores de acordo com segmento corporal (artelhos, tornozelo e pé, tíbia/fíbula/perna, joelho, quadril/fêmur/coxa e alterações sistêmicas de MMII), local de trabalho (administrativo realiza atendimento ao público externo e cuida de questões administrativas internas; e operativo realiza atividades externas em turnos, como por exemplo policiamento à pé, montado, motorizado, em bicicleta, radiopatrulha e atividades típicas da missão), diagnóstico de acordo com a Classificação Internacional de Doenças - $10^{\text {a }}$ revisão (CID-10).

Os dados obtidos foram organizados no programa Microsoft Office Excel 2007 e transferidos para o SPSS 14.0 para Windows, para análise. Todas as variáveis do estudo foram categorizadas e descritas em valores absolutos e em porcentagem. Para identificar a associação entre os fatores: idade, posto, local de trabalho com a DME de MMII foi realizada razão de proporção entre as categorias das variáveis considerando intervalo de confiança de $95 \%$.

\section{Resultados}

Foram analisados 6.687 arquivos de pacientes, provenientes da junta médica da Polícia Militar do Estado da Bahia. Do total de 13.342 CID relacionadas à DME no período de Janeiro de 2003 a Dezembro de 2007, 3.652 apresentavam alguma CID de DME em MMII, sendo sua proporção estimada em 27,5\%.

A maior proporção para o desenvolvimento das doenças em PM se deu a partir de 26 a 34 anos de idade $(30,50 \%)$, em militares praças $(27,80 \%)$, sendo o setor operativo $(27,8 \%)$ o local de trabalho com maior aparecimento das doenças. Os indivíduos nesse setor apresentaram 16 vezes mais possibilidade de adoecerem pelas DME de MMII quando comparados ao setor administrativo, sendo este resultado estatisticamente significante (IC95\%: 10,92- 24,40) (Tabela 1). 
Tabela 1

Proporção de DME em MMII e Outras Doenças de acordo com as variáveis idades, local de trabalho e posto em Policias Militares que procuraram serviço de saúde, Bahia, Brasil, 2003-2007

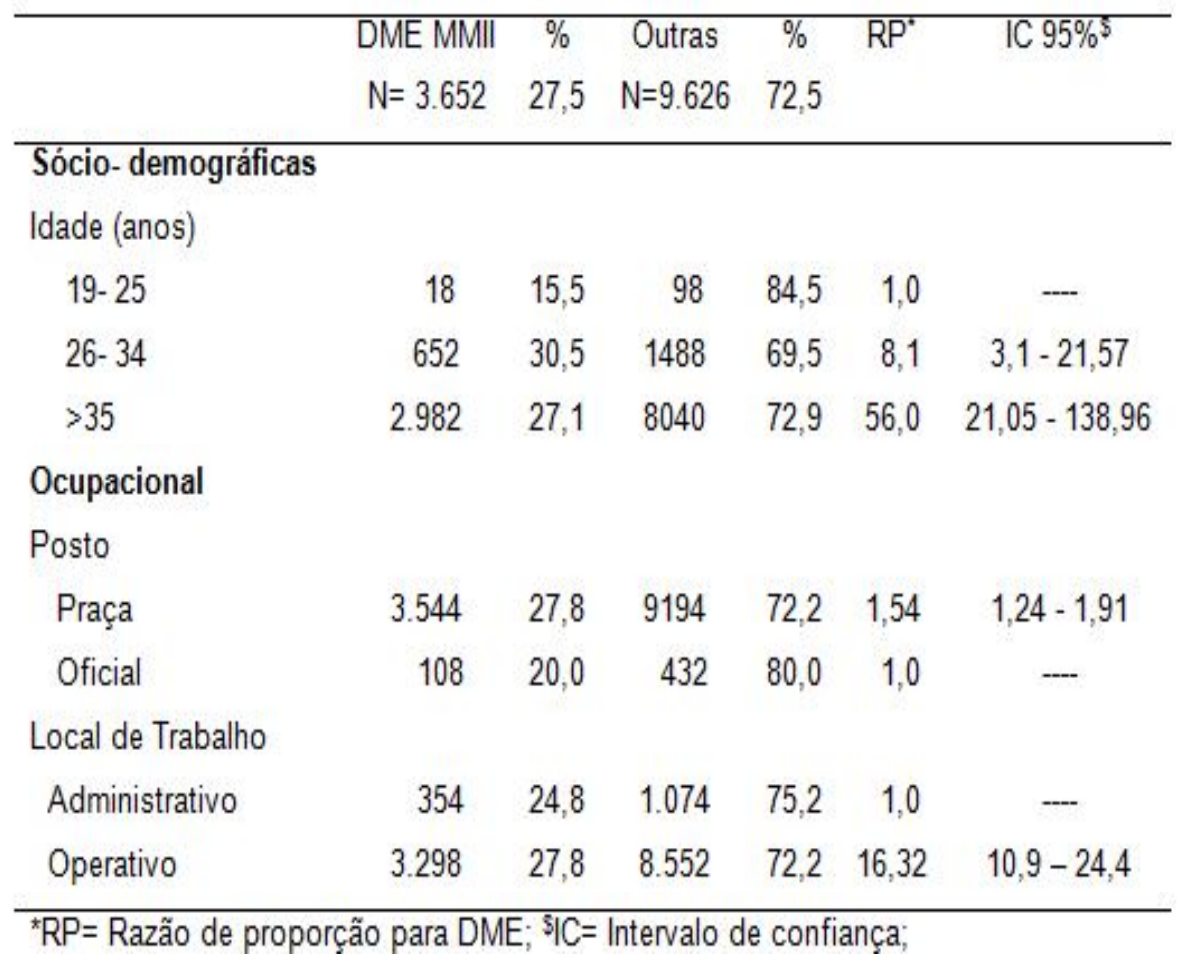

Observou-se que os militares com idade acima de 35 anos apresentaram 56 vezes a mais de possibilidade de desenvolver DME em MMII em relação ao grupo de referência, sendo este resultado estatisticamente significante (IC95\%: 21,05 - 138,96\%). Ainda tratando-se dessa faixa etária verificou-se que as lesões atingem os militares praças em 98,6\% quando comparados com os oficiais $(1,4 \%)$ (Tabela 2$)$.

Tratando-se dos segmentos dos membros inferiores mais afetados pelas DME, foi visto que $45,94 \%$ das lesões ocorreram na região do joelho, seguido de $26,61 \%$ em tornozelo e pé. De acordo com a análise realizada foi observado que os segmentos lesionados não apresentaram diferença em suas proporções, contribuindo de forma semelhante para as DME em membros inferiores. Porém, apesar disso, pode-se verificar a alta proporção desta condição em militares do posto praça, variando de $96,4 \%$ a $97,5 \%$, sendo que os oficiais não apresentaram lesões sistêmicas ou lesões em quadril. 
Tabela 2

Lesões de membros inferiores em Policiais Militares, de acordo com o segmento do corpo, Bahia, Brasil, 2003-2007

\begin{tabular}{|c|c|c|c|c|c|c|c|c|}
\hline $\begin{array}{c}\text { Sócio- } \\
\text { demográficas } \\
\text { Idade (anos) }\end{array}$ & $\begin{array}{c}\text { Pra- } \\
\text { ça } \\
\mathrm{N}= \\
3544\end{array}$ & $\begin{array}{c}\% \\
27,8\end{array}$ & $\begin{array}{c}\text { Ofi- } \\
\text { cial } \\
\mathrm{N}= \\
108\end{array}$ & $\begin{array}{c}\% \\
20,0\end{array}$ & $\begin{array}{c}\text { Total } \\
\mathbf{N}=3.65 \\
3 \\
\mathrm{~N}= \\
\mathbf{3 . 6 5 2}\end{array}$ & $\begin{array}{c}\% \\
27,5\end{array}$ & RP* & IC95\%\$ \\
\hline 19- 25 & 10 & 55,60 & 8 & 44,40 & 18 & 0,4 & 1,0 & ---- \\
\hline $26-34$ & 594 & 91,1 & 58 & 8,9 & 652 & 17,9 & 8,19 & $\begin{array}{l}3,11- \\
21,57\end{array}$ \\
\hline$>35$ & 2.940 & 98,60 & 42 & 1,4 & 2.982 & 81,7 & 56,00 & $\begin{array}{l}21,05- \\
148,96\end{array}$ \\
\hline Local de Trabalho & & & & & $\mathrm{N}=3.646$ & & & \\
\hline Administrativo & 290 & 81,9 & 64 & 18,1 & 354 & 9,7 & 1,0 & ---- \\
\hline Operativo & 3.254 & 98,7 & 44 & 1,3 & 3.292 & 90,3 & 16,32 & $\begin{array}{c}10,92- \\
24,40\end{array}$ \\
\hline $\begin{array}{l}\text { Segmento } \\
\text { Lesionado }\end{array}$ & & & & & $\mathrm{N}=3.652$ & & & \\
\hline Artelhos & & & & & 166 & 4,57 & 1,0 & --- \\
\hline Tornozelo e pé & 948 & 97,5 & 24 & 2,5 & 972 & 26,61 & 0,99 & $0,97-1,03$ \\
\hline $\begin{array}{c}\text { Tíbia/ Fíbula/ } \\
\text { perna }\end{array}$ & 592 & 96,6 & 20 & 3,4 & 592 & 16,21 & 1,40 & $0,48-4,0$ \\
\hline Joelhos & 1.618 & 96,4 & 60 & 3,6 & 1.678 & 45,94 & 1,49 & $0,54-4,0$ \\
\hline $\begin{array}{c}\text { Quadril/ } \\
\text { Fêmur/Coxa }\end{array}$ & 192 & 100 & - & - & 192 & 5,25 & 1.0 & $1,00-1,04$ \\
\hline $\begin{array}{c}\text { Alterações } \\
\text { sistêmicas e de } \\
\text { M M II }\end{array}$ & 52 & 100 & - & - & 52 & 1,42 & 1,0 & $1,00-1,04$ \\
\hline
\end{tabular}

OBS: *RP= Razão de proporção para DME por segmento; \$IC= Intervalo de confiança;

\section{Discussão}

O presente estudo demonstra que embora sejam muitos os indivíduos na Polícia Militar no estado da Bahia que procuram por serviços de saúde, observou-se uma baixa proporção de Doença Músculo Esquelética (DME) em membros inferiores, em que os militares praças, com faixa etária entre 26-39 anos, que ocupam o setor operativo representaram os indivíduos mais susceptíveis. Foi observado que de acordo com o segmento, o joelho foi a região de maior acometimento.

A proporção de DME encontrada nesse estudo foi inferior quando comparada com pesquisas que abordam o tema em militares. Estudos corroboram que a atividade profissional é a principal causa dessas DME, sendo esta o maior motivo de limitação funcional e a segunda maior razão para alta precoce do serviço militar na Finlândia, e seu número vem crescendo fortemente em torno de $62,0 \% 11$. 0 aparecimento da doença nos membros inferiores pode estar relacionado a atividades que exigem exposição repetitiva da região, maior resistência e força física12, esforços mais intensos por períodos prolongados, atividades de grande impacto e altas demandas energéticas13, grandes cargas ou traumas secundários ao seu posto de trabalho, além do processo natural do envelhecimento. Essas características confirmam-se em estudos que mostram que a classe estudada apresenta maior lesão devido às atividades 
inerentes à função militar, onde as corporações destacam-se pela pesada carga de trabalho e sofrimento, além do treinamento que envolve corrida, prática de esporte, carregamento de peso, marcha, movimentos repetitivos, sobreuso, saltos e tiros9.

Um estudo revelou que uma das atividades que leva ao desenvolvimento de DME em militares é a marcha, ocorrendo pela onda de impacto proveniente da força de reação ao solo durante a locomoção, predispondo ao aparecimento de lesões durante o período de adaptação dos indivíduos à vida militar14. Outros estudos com população de enfermeiros e técnicos de enfermagem evidenciaram que a postura em ortostase, por longos períodos representou forte associação para o desenvolvimento de DME em MMII, por conta da sobrecarga ao sistema músculo esquelético15.

A proporção de DME foi maior na faixa etária entre 26-34 anos e acima dessa idade percebeu-se maior possibilidade de desenvolvimento das lesões, corroborando com um estudo referente a lesões esportivas em militares, que aponta as faixas etárias de 19 a 24 anos $(40,0 \%)$ e 25 a 30 anos $(40,0 \%)$, como as que destinaram maiores números de atendimentos por conta de DME16. Estudos em instituições civis e militares observaram que ocorrência de doença musculoesquelética cresce em duas condições cruciais: no início da carreira, quando o trabalhador ainda está se adaptando e adaptando o corpo às demandas das tarefas do trabalho específico e à medida que aumenta a idade, assim como mostra o presente estudo17.

Com o avanço da idade ocorrem modificações de todo o sistema musculoesquelético deixando-o menos resistente aos fatores biomecânicos envolvidos nas demandas físicas que são impostas pelo trabalho militar. A exposição contínua e prolongada dos indivíduos aos fatores como força, repetitividade e altas cargas, tende a sobrecarregar o sistema, o que explica a maior chance de desenvolvimento de DME em MMII na faixa etária observada.

A DME em MMII teve maior proporção em policiais militares praças, corroborando com os resultados encontrados em outros estudos, que colocam o militar praça como o grupo com maior freqüência de lesões musculoesqueléticas entre os anos de 2000 a 2004. Os autores afirmam que o número médio de praças com lesões e traumas músculo esquelético cresceu $227,5 \%$. Inicialmente representou 13 vezes mais, quando comparados com os oficiais passando a ser 16.8 vezes em 20049.

Conforme disposto na Lei no 6.783 de 16 de outubro de 1974 sobre o Estatuto dos Policiais Militares do Estado de Pernambuco, Seção II - Do Comando e Da Subordinação (PERNAMBUCO, 1974) na hierarquia, o Oficial é preparado para exercer cargos de chefia e comando, e a estes se subordinam os praças18. Sendo assim os oficiais enquadram-se em um setor mais administrativo e os praças trabalham em setor mais operativo, isso pode justificar o fato da maior vulnerabilidade para lesões, já que os praças passam a estar mais expostos aos fatores lesivos, podendo sofrer por lesões agudas e traumáticas por encontrarem-se na linha de frente dos confrontos. Nesse posto, os policiais convivem com jornadas perigosas e prolongadas, em turno noturno, com atividades de risco, equipamentos precários, além da disciplina conferidas pela organização do trabalho militar19. De acordo com o estudo, os militares praças configuram $93 \%$ dos incapacitados físicos retirados dos serviços ostensivos para realizar tarefas internas9.

Pode-se observar no presente estudo uma proporção 16 vezes mais elevada no setor operativo para o desenvolvimento de DME em MMII, quando comparado com o setor administrativo, intensificando os motivos já citados anteriormente com relação à maior exposição dos militares no setor operativo.

0 presente estudo apontou o joelho como o principal segmento dos membros inferiores afetados pela DME seguido pelo tornozelo e pé, semelhantemente a estudos com soldados, onde foram agrupadas as articulações mais acometidas, sendo o tornozelo com $58 \%$ e o joelho com $25,8 \%$ das lesões 20,21 . Outras pesquisas referiram também ambas as regiões como principais acometidas, como em um estudo envolvendo lesões esportivas em militares, em que o tornozelo teve maior acometimento $(60,0 \%)$ seguido do joelho $(40,0 \%) 16$. Em outro estudo relacionado ao treinamento físico militar, os autores demonstraram que 44\% (22\% 
tornozelo e $22 \%$ joelho) das lesões ocorrem nessas regiões, onde a incidência apresentou-se em porcentagens iguais21.

Todas estas lesões podem ser justificadas pelo treinamento físico militar, como também pelos métodos de treino incorretos, alterações estruturais que forçam certas partes do corpo mais do que outras e fraqueza dos músculos, tendões e ligamentos. Estas lesões podem exceder a capacidade de recuperação tecidual, levando a um ciclo vicioso de irritação inflamatória local, retenção de metabolitos, dor, isquemia e edema20.

Este estudo apresenta limitações de cunho científico, pela escassez de publicações relacionadas à saúde física do policial militar e principalmente às lesões músculo-esqueléticas decorrentes da sua ocupação, representando a falta de atenção à saúde do policial enquanto trabalhador. Existe ainda uma limitação inerente à fonte de dados secundária, que não permitiu aprofundar as questões específicas relacionadas à saúde prévia dos militares ou mesmo detalhamento sobre a ocupação, carga horária de trabalho, e dados específicos de exposição (movimentos repetitivos, posturas, vestuário, carga horária de trabalho, tempo de trabalho como policial militar, entre outros).

\section{Conclusão}

A proporção de DME de MMII em policiais militares da Bahia foi considerada baixa. Apesar dos resultados obtidos, sugerem-se estudos futuros com o intuito de dar visibilidade à problemática em relação à saúde dos policiais militares em nível nacional, bem como políticas de prevenção, como uma forma de prevenir e/ou diminuir a ocorrência das DME em membros inferiores na população estudada.

\section{Referências}

1. WOOLF, A.D. et al. "Burden of major musculoskeletal conditions", Bulletin of the World Health Organization, 2003.

2. Brasil. Ministério da Saúde. Diagnóstico, tratamento reabilitação, prevenção e fisiopatologia das LER/ DORT, Série A. Normas e Manuais Técnicos, \# 105, 2001.

3. Brasil. Ministério da Saúde. LER/DORT: dilemas, polêmicas e dúvidas. Série A. Normas e Manuais Técnicos, \# 104, 2001.

4. WALSH, IAP et al. "Capacidade para o trabalho em indivíduos com lesões musculoesqueléticas crônicas", Revista de Saúde Pública, 38(2): 149-56. 2004.

5. SOUZA, N. S. S; SANTANA, V. S. "Incidência cumulativa anual de doenças musculoesqueléticas incapacitantes relacionadas ao trabalho em uma área urbana do Brasil", Cadernos de Saúde Pública, 2124-34. 2011.

6. FRAGA, C. K. "Peculiaridades do trabalho policial militar", Revista Virtual Textos \& Contextos, (6): 2006.

7. MINAYO, M. C. S; ASSIS, S. G; OLIVERIA, R. V. C. "Impacto das atividades profissionais na saúde física e mental dos policiais civis e militares do Rio de Janeiro (RJ, Brasil)", Revista Ciência \& Saúde Coletiva, 2: 1990-2209. 2011.

8. WHITE, M. R, MCNALLY, M. S. "Morbidity and mortality in U.S. Navy personnel from exposures to hazardous materials", Military Medicine, 70-73. 1991.

9. SOUZA, E. R, MINAYO, M. C. S. "Policial, risco como profissão: morbimortalidade vinculada ao trabalho", Revista Ciência e Saúde Coletiva, 10(4): 917-28. 2005.

10.SANTOS, E. C, MARTINS, V. F. Lombalgia: Custo, absenteísmo e fatores associados em Policiais Militares do Estado da Bahia. Salvador, 2009.

11. TAANILA, H. "Musculoskeletal disorders in physically active conscripts: a one-year follow-up study in the Finnish Defence Forces", BMC Musculoskeletal Disorders, 2009. 
12. NEVES E. B. "Gerenciamento do risco ocupacional no Exército Brasileiro: aspectos normativos e práticos", Rio de Janeiro-RJ: Caderno de Saúde Pública, 23(9): 2127-33. 2007.

13.SILVA, F.; SILVA, M. Lesão em joelho de militares brasileiros e os fatores associados. EBMSP [Trabalho de conclusão de curso para obtenção do título de Bacharel em Fisioterapia, Escola Bahiana de Medicina e Saúde Pública, Salvador, 2011].

14.GUISANDE, T.P, MOCHIZUKI, L. "Forças de impacto e marcha militar: estudo descritivo", Revista de Educação e de Tecnologia Aplicadas à Aeronáutica, 1: 61-67. 2009.

15. RIBEIRO, N. F, FERNANDES, R. C. P. “Distúrbios Musculoesqueléticos em membros inferiores em trabalhadoras de enfermagem", Revista Baiana de Saúde Pública, 35(1): 128-42. 2011.

16. SOUSA, M. S. C et. al. “Epidemiologia e Saúde: prevalência das lesões musculares esqueléticas (LME) esportivas em instituições cíveis e militares (Exército Brasileiro) da cidade de João Pessoa", Revista Brasileira de Ciência e Movimento, 12(1): 45-50. Jan-Mar/ 04.

17. MONTEIRO, M.S et al. "Doenças músculo-esqueléticas, trabalho e estilo de vida entre trabalhadores de uma instituição pública de saúde”, Revista da Escola de Enfermagem, 40(1): 20-25. 2006.

18. Estatuto dos Policiais Militares do Estado de Pernambuco, Seção II - Do Comando e Da Subordinação (Pernambuco, 1974).

19. BRAZ, D.M. A Saúde do Policial Militar no Rio Grande do Sul: acidente de trabalho na atividade física e treinamento. [Tese apresentada à Faculdade de Serviço Social - PUCRS, como requisito parcial para a obtenção do grau de Mestre em Serviço Social do Programa de Pós-Graduação em Serviço Social da Pontifícia Universidade Católica, Porto Alegre, 2005].

20.TEODORO, H. C; ROSAS, R. F. Prevalência de lesões músculo esqueléticas no treinamento físico militar do $63^{\circ}$ batalhão de infantaria de Tubarão - SC. [ Trabalho de conclusão de curso para obtenção de título de Bacharel em Fisioterapia. Universidade do Sul de Santa Catarina, Tubarão].

21.GONÇALVES, E. M, SILVA, R. R. Principais Lesões Decorrentes do Treinamento Físico Militar no Centro Integrado de Guerra Eletrônica - Departamento de Ciência de Tecnologia do Exército Brasileiro. \# 203, 2009. 